\title{
Hazard rate of tumor recurrence over time in patients with colon cancer: implications for postoperative surveillance from three Japanese Foundation for Multi- disciplinary Treatment of Cancer (JFMC) clinical trials
}

\author{
1. Cancer Treatment Center, Kochi Medical School Hospital, Kochi University, Nankoku, Japan \\ 2. Department of Biostatistics, Graduate School of Medicine, The University of Tokyo, Tokyo, Japan \\ 3. Department of Surgery, Yokohama City University, Yokohama, Japan \\ 4. Department of Minimally Invasive Surgical and Medical Oncology, Fukushima Medical University, Fukushima, Japan \\ 5. Department of Surgery, Keio University School of Medicine, Tokyo, Japan \\ 6. Department of Gastroenterological Surgery (Surgery II), Nagoya University Graduate School of Medicine, Nagoya, Japan \\ Faculty of Engineering, Tokyo University of Science, Tokyo, Japan \\ Department of Surgery, Tokai University, Isehara, Japan \\ 9. Tokai Central Hospital, Kakamigahara, Japan \\ 10. Japanese Foundation for Multidisciplinary Treatment of Cancer, Tokyo, Japan \\ 11. Department of Gastrointestinal Surgery, Kanagawa Cancer Center, Yokohama, Japan \\ *These authors equally contributed to this study.
}

Hiromichi Maeda ${ }^{* \bowtie}$, Kosuke Kashiwabara ${ }^{2 *}$, Toru Aoyama ${ }^{3}$, Koji Oba ${ }^{2}$, Michitaka Honda ${ }^{4}$, Shuhei Mayanagi ${ }^{5}$, Mitsuro Kanda ${ }^{6}$, Chikuma Hamada7, Sotaro Sadahiro ${ }^{8}$, Junichi Sakamoto ${ }^{9}$, , Shigetoyo Saji ${ }^{10}$, Takaki Yoshikawa ${ }^{11 \bowtie}$

$\triangle$ Corresponding authors: Hiromichi Maeda, MD, PhD, Cancer Treatment Center, Kochi Medical School Hospital, Kochi University, Kohasu, Oko-cho, Nankoku, Kochi, 783-8505, Japan E-mail; hmaeda@kochi-u.ac.jp, Telephone: +81-88-880-2370, Fax: +81-88-880-2371 and Takaki Yoshikawa, MD, PhD, Department of Gastrointestinal Surgery, Kanagawa Cancer Center, Yokohama 241-8515, Japan E-mail; yoshikawat@kcch.jp, Telephone: +81-45-520-2222, Fax: +81-45-520-2202

(C) Ivyspring International Publisher. This is an open access article distributed under the terms of the Creative Commons Attribution (CC BY-NC) license (https://creativecommons.org/licenses/by-nc/4.0/). See http://ivyspring.com/terms for full terms and conditions.

Received: 2017.06.07; Accepted: 2017.09.30; Published: 2017.10.23

\begin{abstract}
Purpose: Reliable risk estimates of recurrence are necessary to establish optimal postoperative surveillance strategies. The purpose of the present study was to clarify changes in the hazard rate $(H R)$ for tumor recurrence over time in Japanese patients with colon cancer.

Methods: Data for 3984 patients from three clinical trials evaluating the benefit of adjuvant chemotherapy for colon cancer were analyzed. Estimated HRs were plotted over time for the entire cohort, as well as for node-positive and node-negative patients separately. The changes in risk were further analyzed according to eight clinical variables, and factors predictive of early ( $<3$ years) and late (>3 years) recurrence were explored using Cox's regression analysis.

Results: In node-positive patients, there was a prominent HR peak 0.6 years after surgery, whereas HR remained at consistently low levels in node-negative patients. In node-positive patients, HR decreased steadily until 3 years, after which the decline in HR plateaued. Those with T4 tumors had a prominent HR peak around 1 year, including node-negative patients. The HR for T1/T2 Stage III colon cancers showed a similar pattern as that for T1-T3 node-negative colon cancers. Cox regression analysis revealed that a lack of adjuvant chemotherapy, positive node status, T3/T4 factors, and male gender predict early recurrence, whereas patients with lymph node metastasis, T4 tumors, and a lesser extent of lymph node removal have a higher risk of recurrence $3-4$ years after surgery $(P<0.05)$.

Conclusion: The present study supports the concept of intensive surveillance during the first 3 years after curative resection. However, a reduction in surveillance intensity may be acceptable for patients with T3 Stage II and T1/T2 Stage III colon cancer.
\end{abstract}

Key words: pooled analysis, continuous-time hazard, node-positive patients, T1, T2, intensive surveillance 


\section{Introduction}

In 2013, 1.6 million colorectal cancers were newly diagnosed worldwide, and more than 0.7 million patients died of disease progression [1]. In patients with localized disease, wide surgical resection followed by adjuvant chemotherapy results in a 5 -year disease-free survival (DFS) rate of $>70 \%[2,3]$. Chemotherapy and surgical resection of recurrent metastatic tumors of colorectal cancers still offer a chance of cure [4,5], and advances in such treatments strengthen the paradigm that the early detection of treatable recurrence through appropriate postoperative surveillance benefits patients [6]. At the same time, the surveillance schedule has to be strictly adhered to by patients for the expected benefits to be realized, and this requires a clear explanation to be given to patients, with efforts made to avoid unnecessary examinations.

Statistical risk analysis of tumor recurrence forms the basis of an optimal surveillance schedule. More than $80 \%$ of recurrences occur within the first 3 years [7, 8], and the guidelines of the American Society of Clinical Oncology recommend conducting intensive surveillance in the first 2-4 years for patients with Stage II/III tumors [9]. Although the appropriate interval for postoperative testing remains contentious, clinicians may tend towards more frequent examinations for patients at higher risk of recurrence [9]. Consensus guidelines from the European Society for Medical Oncology also endorse frequent clinical visits during the first 3 years, with computed tomography performed annually or 6 monthly [10]. In addition, nomograms have been proposed to estimate the risk of the tumor recurrence within a certain period of time based on clinical variables [11]. Information obtained from risk calculations via nomograms is expected to improve individuals' understanding of the disease and consequently enhance their adherence to treatment and surveillance.

As the next step in setting an evidence-based timetable for postoperative surveillance, we consider it important to understand changes in the risk of recurrence over time, and to determine how clinical factors affect these changes. This information would enable identification of patients who would obtain a clinical benefit through intensive monitoring more specifically, and would inform decisions regarding the continuation or discontinuation of surveillance. Thus, in the present study, chronological change in estimated hazard rates (HRs) were delineated using data from three independent clinical trials for adjuvant chemotherapy [12-15]. It was anticipated that using data from nearly 4000 patients who underwent follow-up examinations according to regimens determined in each study separately would yield meaningful information. Further analysis taking into consideration eight clinical variables of interest was performed to determine how these variables affect changes in the risk of recurrence in node-negative and node-positive colon cancer. Finally, the risk factors for early ( $<3$ years) and late (3-5 years) recurrence were explored statistically to support the interpretation of HRs.

\section{Methods}

\section{Clinical trials and patients}

The present study used data from three clinical trials performed by the Japanese Foundation for Multidisciplinary Treatment of Cancer (JFMC) [12-15]. These trials were conducted to elucidate the clinical efficacy of fluoropyrimidine-based adjuvant chemotherapy with different treatment schedules, with or without additional therapeutic agents.

The aim of JFMC Project no. 07-8601 (JFMC07-8601) was to determine the clinical benefit of combination adjuvant chemotherapy with mitomycin $\mathrm{C}$ and oral fluoropyrimidines or 5-fluorouracil intravenous bolus in curatively resected colon and rectal cancer [15]. The combination adjuvant treatment yielded statistically significant improvements in DFS in patients with rectal cancer, although this effect was not evident in patients with colon cancer. JFMC Project no. 15-8901 (JFMC15-8901) was performed to elucidate the safety and efficacy of adjuvant immunochemotherapy using fluoropyrimidines and OK-432 (a streptococcal agent), but failed to demonstrate any survival benefit of this treatment regimen [13, 14]. These two clinical trials included patients allocated to surgery alone, with the comparative analysis of adjuvant chemotherapy versus surgery alone proving the benefit of adjuvant chemotherapy on DFS [3] in both colon and rectal cancer.

JFMC Project no. 33-0502 (JFMC33-0502) was conducted to determine the optimal schedule for adjuvant chemotherapy with oral fluoropyrimidines and leucovorin (uracil-tegafur plus leucovorin) in curatively resected Stage IIB and III colon cancer [12]. The main finding of that trial was that a longer duration of treatment (18 months) did not improve overall survival or DFS compared with shorter treatment (6 months), and the longer duration of adjuvant chemotherapy with this regimen was not recommended [12].

The protocols of all three trials were approved by each of the institutes in which the trials were performed, and treatment was initiated after patients 
had provided written informed consent. Approval for conducting a pooled analysis of the three trials [16] was obtained from the Internal Review Board of the JFMC. Because of anatomical differences and differences in the pattern of recurrence between colon and rectal cancers, the present study excluded rectal cancers from analysis. Thus, the present study included data from 3984 patients with curatively resected adenocarcinoma from the cecum to the rectosigmoid junction (see Figure S1, available as Supplementary Material to this paper).

\section{Data preparation and statistical analysis}

Node status in JFMC07-8601 and JFMC15-8901 was recorded according to the 3rd and 4th editions of the Japanese classification of colorectal carcinoma issued by the Japanese Research Society for Cancer of Colon and Rectum [17, 18], in which the extent of node involvement was classified according to the location of the metastasis. Therefore, it was not possible to convert node status into $\mathrm{N}$ factors of the UICC-TNM classification of malignant tumors, and node status was simply dichotomized as positive or negative. Well- and moderately differentiated adenocarcinomas were grouped as low-grade tumors, whereas mucinous adenocarcinoma, poorly differentiated adenocarcinoma, and signet-ring cell carcinoma were grouped as high-grade tumors. Although the agents used and treatment schedules differed across the three trials, in the present study patients were divided into two categories: (i) those undergoing surgery alone, and (ii) those undergoing surgery with adjuvant therapy. The extent of lymphadenectomy was also defined according to the Japanese Classification of Colorectal Carcinoma, the basic concept of which has remained unchanged across editions. Conceptually, D3 dissection of lymph nodes consists of en bloc removal of lymph nodes along the feeding artery, as well as the distant draining lymph nodes [19]. D2 dissection involves removal of the lymph nodes from the intestine to the root of the direct feeding artery. The outcome in the present study was DFS, which was defined as the time from the date of surgery to either tumor recurrence or death due to any cause.

HR plots were constructed for all patients, as well as for node-positive and node-negative patients separately because node status is the most significant determinant of recurrence after curative resection of colon cancer. Further assessment of changes in the risk of recurrence was performed by stratifying patients according to clinical variables of interest, namely gender, age, $\mathrm{T}$ factors, tumor grade, adjuvant chemotherapy, presence of postoperative complications, tumor site, and extent of regional lymph node resection. This method allows the visual assessment of the chronological change of risk for tumor recurrence. The plot of HR doesn't require any distributional assumption, which is a critically different point from Cox proportional hazards regression models [2].

To determine factors predictive of early and late recurrence, Cox regression analysis was performed. The predictive factors included in the models were chosen by the backward selection method with the inclusion criterion of $P<0.2$. Finally, factors with $P<$ 0.05 were identified as independent factors affecting either early or late recurrence. All analyses were performed using the LIFETEST and PHREG procedures in SAS 9.4 (SAS Institute, Cary, NC, USA). The explanatory notes were added by using Photoshop CS2 (Adobe Systems, San Jose, CA, USA).

\section{Results}

\section{Patient characteristics}

Of the 3984 patients, $55.7 \%$ were aged $\geq 60$ years, $54 \%$ were male, $46.3 \%$ had node-negative cancers, $68.6 \%$ underwent adjuvant chemotherapy, and $62.1 \%$ underwent D3 lymph node dissection; $15 \%$ of patients had postoperative complications, $6.0 \%$ of tumors were high grade, and $10.4 \%$ of cancers were of the transverse colon.

\section{Changes in risk of recurrence over time}

$H R$ of recurrence for all patients and node-positive and -negative tumors

Plotting HR over time revealed that the risk of recurrence increased steeply towards a peak $(\mathrm{HR} \approx$ $0.1) 0.8$ years after resection, after which it decreased to less than half the peak at 3 years (Figure 1 ).

Having lymph node metastasis increased the risk of tumor recurrence within the first 3 years. The HR of recurrence for node-positive colon cancers was highest around 0.6 years after surgery (Figure 1B), with much higher maximum $H R$ values than for node-negative colon cancer. In node-negative colon cancer, the HR peak was observed soon after 1 year, with a value of $0.05-0.06$. Notably, this value of HR is similar to that for node-positive colon cancers 2.5-3 years after surgery. Although the HR for node-positive colon cancer decreased gradually until 3 years after surgery, it remained higher than that for node-negative colon cancers at least up to 5 years (Figure 1B).

\section{Effects of clinical variables on the HR for the entire patient cohort}

Patients were divided into groups according to clinical variables of interest, and HRs were plotted 
against time. This analysis offered visual information regarding the effects of clinical variables on changes in HRs, although the effects of confounders were not overcome.

As expected, patients with T4 or T3 colon cancer had greater risks of recurrence, especially early after surgery (Figure 2A). Peak HR for both T3 and T4 tumors was observed around 0.8 years after surgery, with values of approximately 0.16 and 0.08 , respectively. In contrast, peak HR for T1/T2 tumors was as late as 1.5 years after surgery, with a value of around 0.04 . Overall, in patients undergoing adjuvant chemotherapy, the HR appeared to be lower during the first 2.5 years after surgery (Figure 2B). However, thereafter the risk of recurrence was similar for patients undergoing surgery with or without adjuvant chemotherapy. The HR was higher for male than female patients (Figure 2C). However, the effects of D2 versus D3 lymph node dissection were minimal when the entire patient population was analyzed (Figure 2D).

Further plots were created using another four variables (see Figure S1). Dividing patients into groups based on pathological tumor type revealed that tumor recurrence early after operation was higher for patients with high-grade versus low-grade tumors. However, the HR values crossed several times, probably due to the relatively small number of patients in these groups (Table 1; Figure S1A). A similar trend was observed for HR plots of patients with versus without postoperative complications (see Figure S1B). Cancers in the ascending colon (versus left-side colon cancer) seemed to be associated with a greater chance of recurrence during the first 2 years after surgery (see Figure S2C). Finally, tumor recurrence was more frequent in older $(>60$ years) than younger patients, although the contours of the HR plots were similar (see Figure S2D).

Effects of clinical variables on HRs in node-positive and -negative disease

The effects of each clinical variable on changes in HR were assessed after patients had been divided into node-positive and node-negative groups.

The extent of wall invasion had a significant effect on prognosis, with T4 node-positive (Stage III) disease having the highest HR peak compared with the other groups (Figure 3A). There was no prominent HR peak for T2/T1 disease, even in patients with lymph node metastasis, and the HR values for node-positive T2/T1 and node-negative T3 tumors were low and consistent across time (Figure 3A). Importantly, HR in 
node-negative patients with $\mathrm{T} 4$ disease peaked 1 year after surgery, with a maximum of approximately 0.08 . Although the HR in this group decreased gradually over 2 years, it remained higher than the HR for $\mathrm{T} 1 / \mathrm{T} 2$ node-positive colon cancers (Figure $3 \mathrm{~A}$ ).

Table 1. Clinical variables

\begin{tabular}{|c|c|c|c|c|}
\hline & JFMC07-8601 & JFMC15-8901 & JFMC33-0502 & Total \\
\hline \multicolumn{5}{|l|}{ Age (years) } \\
\hline$<60$ & 856 & 554 & 354 & 1764 (44.3) \\
\hline$\geq 60$ & 834 & 676 & 710 & $2220(55.7)$ \\
\hline \multicolumn{5}{|l|}{ Gender } \\
\hline Male & 937 & 661 & 554 & $2152(54.0)$ \\
\hline Female & 753 & 569 & 510 & $1832(46.0)$ \\
\hline \multicolumn{5}{|l|}{ Node status } \\
\hline Positive & 537 & 385 & 921 & $1843(46.3)$ \\
\hline Negative & 1153 & 845 & 143 & $2141(53.7)$ \\
\hline \multicolumn{5}{|l|}{$\mathrm{T}$ factor } \\
\hline $\mathrm{T} 1 / \mathrm{T} 2$ & 206 & 134 & 128 & 468 (11.8) \\
\hline T3 & 910 & 966 & 554 & $2430(61.0)$ \\
\hline $\mathrm{T} 4$ & 574 & 130 & 382 & $1086(27.3)$ \\
\hline \multicolumn{5}{|c|}{ Postoperative complications } \\
\hline Positive & 370 & 176 & 81 & 627 (15.7) \\
\hline Negative & 1320 & 1054 & 983 & 3357 (84.3) \\
\hline \multicolumn{5}{|c|}{ Tumor grade ${ }^{A}$} \\
\hline High & 91 & 67 & 79 & $237(6.0)$ \\
\hline Low & 1599 & 1163 & 985 & $3747(94.1)$ \\
\hline \multicolumn{5}{|c|}{ Adjuvant chemotherapy } \\
\hline Yes & 836 & 834 & 1064 & $2734(68.6)$ \\
\hline No & 854 & 396 & 0 & $1250(31.4)$ \\
\hline \multicolumn{5}{|c|}{ Tumor location } \\
\hline $\begin{array}{l}\text { Ascending } \\
\text { colon }\end{array}$ & 392 & 300 & 318 & $1010(25.4)$ \\
\hline $\begin{array}{l}\text { Transverse } \\
\text { colon }\end{array}$ & 180 & 138 & 98 & $416(10.4)$ \\
\hline Left side ${ }^{B}$ & 1118 & 792 & 948 & 2558 (64.2) \\
\hline \multicolumn{5}{|c|}{ Lymph node dissectionc } \\
\hline $\mathrm{D} 2$ & 732 & 496 & 282 & $1510(37.9)$ \\
\hline D3 & 958 & 734 & 782 & 2474 (62.1) \\
\hline \multicolumn{5}{|c|}{$\begin{array}{l}\text { Data show the number of patients in each group, with percentages given in } \\
\text { parentheses in the Total column. }\end{array}$} \\
\hline \multicolumn{5}{|c|}{$\begin{array}{l}\text { ATumors were divided pathologically into two categories (see text for details). } \\
\text { BLeft side colon, including the ascending colon, sigmoid colon, and rectosigmoid } \\
\text { colon. }\end{array}$} \\
\hline $\begin{array}{l}{ }^{C} \text { For details of } \\
\text { extent of D3 di }\end{array}$ & 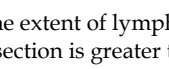 & $\mathrm{h}$ node dissection & , refer to text. Ho & wever, the \\
\hline
\end{tabular}

In the present study, the benefit of adjuvant chemotherapy seemed more evident in node-negative colon cancer (Figure 3B). As for analyses of the entire patient cohort, the effect of adjuvant chemotherapy was limited to within the first 2 years in node-negative patients. Meanwhile, in node-positive patients, the suppression of tumor recurrence by adjuvant chemotherapy seemed marginal.

Male gender seemed to have a more significant effect on prognosis in node-positive colon cancers, especially during the first 3 years after surgery (Figure 3C). Investigating the relationship between node status and lymph node dissection revealed that D3 lymph node dissection had a significant effect on the HR for node-positive tumors 0.6-2 years after surgery, and then again after 3 years (Figure 3D).

Further analysis performed using another four clinical variables revealed considerable fluctuations in the plots due largely to the relatively small number of patients within each of these groups (see Figure S3).

\section{Multivariate analysis}

Factors predicting recurrence within the first 3 years

All clinical variables (eight variables and lymph node status) set before the present study was started were chosen using the backward selection method with the inclusion criterion of $P<0.2$ (Table 2). Lymph node metastasis and T factors (T3/T4) increased the risk of early recurrence by more than double (Hazard ratio $=2.513$ for positive lymph node status; Hazard ratio $=2.09$ for T3 disease; Hazard ratio $=3.166$ for $\mathrm{T} 4$ disease). Meanwhile, the effects of adjuvant chemotherapy and gender were moderate, with both decreasing early recurrence by $14.8 \%$ and $17 \%$, respectively.

\section{Factors predicting recurrence between 3 and 5 years}

The same approach as described above was used to determine the factors affecting tumor recurrence 3-5 years after surgery. The effects of node status and $\mathrm{T}$ factors were less significant on late than early recurrence. Positive node status and T factors (T3 and T4 disease) increased the chance of tumor recurrence by $56 \%, 64 \%$, and $89 \%$, respectively. The extent of lymph node dissection affected late tumor recurrence, whereas adjuvant chemotherapy did not reduce the risk of recurrence any further during this period.

\section{Discussion}

Risk analysis of tumor recurrence is indispensible for detection of treatable recurrence after curative resection of colon cancer while avoiding unnecessary examinations. In this study, the HR of recurrence increased steeply until approximately 0.8 years after surgery, decreasing gradually thereafter. Three years after surgery, the HR had fallen to half its maximum level. From another viewpoint, in the present study $65.3 \%$ and $82.9 \%$ of recurrences had accumulated within 2 and 3 years (data not shown), respectively, which is comparable with rates reported previously $[7,8]$. These findings support current recommendations of intensive surveillance within the first few years after resection. Meanwhile, the HR for recurrence in node-negative colon cancers did not show a significant peak within 5 years after resection, with a maximum HR similar to that seen between 2.5 and 3.5 years after resection of node-positive colon cancers. Although many guidelines for colorectal cancer discuss postoperative surveillance without distinguishing between Stage II and III disease, less intensive surveillance may be acceptable for Stage II colon cancer, as suggested previously [2]. 
Further analysis after stratifying patients according to node status provided more information concerning the surveillance strategy. First, the HR for recurrence of T1/T2 node-positive patients did not have an apparent peak and was maintained at a flat and relatively low level. Second, T4 node-negative cancers had a higher risk of recurrence at around 1 year after resection than T1/T2 nodepositive colon cancers. Although the risk of recurrence for all Stage II colon cancers is much smaller than that for all Stage III cancers, the findings of the present study suggest that patients with T4 node-negative colon cancer need more intensive surveillance. Accordingly, reduced surveillance intensity may be justified for T1/T2 node-positive Stage III colon cancer and T3 Stage II colon cancer. This suggestion is in accordance with the results of Cox regression analysis of factors predicting early recurrence.

The present study is unique in that it explored the risk factors for late recurrence (3-5 years after resection). This analysis revealed that patients with node-positive colon cancer and T4 tumors were still at a higher risk of recurrence during the late phase of surveillance. In addition, patients who underwent lymph node dissection to a lessor extent have a significantly higher risk of recurrence. According to the HR plots, the effects of D3 dissection seem more pronounced in Stage III colon cancer. The clinical benefit derived from continuing intensive surveillance for patients with these independent risks is not known. However, considering the risk of recurrence in patients with $\mathrm{T} 1 / \mathrm{T} 2$ node-positive and T3 node-negative colon cancer within 3 years of surgery, continuation of intensive surveillance for patients with these clinical variables seems sensible.

The present study has several limitations. First, the study was performed using data from three clinical trials that examined various kinds and lengths of fluoropyrimidine-based adjuvant chemotherapy. These studies did not demonstrate significant differences in clinical benefit between different treatments [12-15]. Thus, the effects of the different regimens on the changes in HR over time should also be minimal. However, had the treatment regimens in the three trials been more similar, it may have been easier to clarify the effects of other variables on changes in the risk of recurrence. Second, it was not possible to translate node status into the UICC-TNM classification, and so node status was simply dichotomized into negative and positive. If the UICC-TNM classification had been available, this would have deepened our analysis and helped illustrate how to create a more personalized postoperative surveillance scheme. Third, oxaliplatin-based combination therapy has become the primary adjuvant chemotherapy for colon cancer, and its effects on HR have to be clarified in the Japanese population. In particular, the benefits of adding oxaliplatin seem to be evident in colon cancer with a poorer prognosis, such as $\mathrm{T} 4$ disease and N2b disease [2]. Nevertheless, oral fluoropyrimidines remain treatment options in Japan [20, 21], especially for patients with Stage IIIA colon cancer, in whom the additional benefit seems marginal in the face of toxicities, or for patients too frail to receive doublet chemotherapy.

Figure 3. Hazard rate $(H R)$ for tumor recurrence over time for node-positive (N+; solid lines) and node-negative ( $N$-; dashed lines) with stratification according to (A) T factors, (B) adjuvant chemotherapy or surgery alone, (C) gender, and (D) degree of lymph node dissection. 
Table 2. Cox regression model analysis for prediction of early tumor recurrence

\begin{tabular}{|c|c|c|c|c|c|}
\hline Variables & & $\begin{array}{l}\text { Estimate } \\
\text { (SE) }\end{array}$ & $\begin{array}{l}\text { Hazard } \\
\text { ratio }\end{array}$ & $95 \%$ CI & $P$-value \\
\hline Node status & $\begin{array}{l}\text { Positive (vs. } \\
\text { negative) }\end{array}$ & $\begin{array}{l}0.92134 \\
(0.07919)\end{array}$ & 2.513 & $\begin{array}{l}2.151-2 . \\
935\end{array}$ & $<.0001$ \\
\hline \multirow[t]{2}{*}{$\mathrm{T}$ factor } & T3 (vs. T2/T1) & $\begin{array}{l}0.73711 \\
(0.16802)\end{array}$ & 2.090 & $\begin{array}{l}1.504-2 . \\
905\end{array}$ & $<.0001$ \\
\hline & T4 (vs. T2/T1) & $\begin{array}{l}1.15236 \\
(0.17229)\end{array}$ & 3.166 & $\begin{array}{l}2.258-4 . \\
437\end{array}$ & $<.0001$ \\
\hline $\begin{array}{l}\text { Adjuvant } \\
\text { therapy }\end{array}$ & Yes (vs. no) & $\begin{array}{l}-0.15969 \\
(0.08128)\end{array}$ & 0.852 & $\begin{array}{l}0.727-1 . \\
000\end{array}$ & 0.0495 \\
\hline Gender & Male (vs. female) & $\begin{array}{l}0.16323 \\
(0.07533)\end{array}$ & 1.177 & $\begin{array}{l}1.016-1 . \\
365\end{array}$ & 0.0302 \\
\hline Age & $\begin{array}{l}\geq 60 \text { years (vs. }<60 \\
\text { years) }\end{array}$ & $\begin{array}{l}0.14509 \\
(0.07541)\end{array}$ & 1.156 & $\begin{array}{l}0.997-1 . \\
340\end{array}$ & 0.0543 \\
\hline Tumor grade $\mathrm{A}$ & Low (vs. high) & $\begin{array}{l}-0.22072 \\
(0.14021)\end{array}$ & 0.802 & $\begin{array}{l}0.609-1 . \\
056\end{array}$ & 0.1154 \\
\hline Complication & Yes (vs. no) & $\begin{array}{l}0.13746(0 . \\
0990)\end{array}$ & 1.147 & $\begin{array}{l}0.945-1 . \\
393\end{array}$ & 0.1650 \\
\hline \multirow[t]{2}{*}{ Tumor location } & $\begin{array}{l}\text { Left side }^{B} \text { (vs. } \\
\text { A-colon) }\end{array}$ & $\begin{array}{l}-0.13823 \\
(0.08479)\end{array}$ & 0.871 & $\begin{array}{l}0.738-1 . \\
028\end{array}$ & 0.1030 \\
\hline & $\begin{array}{l}\text { T-colon (vs. } \\
\text { A-colon) }\end{array}$ & $\begin{array}{l}-0.20106 \\
(0.13829)\end{array}$ & 0.818 & $\begin{array}{l}0.991-1 . \\
334\end{array}$ & 0.1460 \\
\hline $\begin{array}{l}\text { Lymph node } \\
\text { dissectionc }\end{array}$ & D2 (vs. D3) & $\begin{array}{l}0.13959 \\
(0.07598)\end{array}$ & 1.150 & $\begin{array}{l}0.624-1 . \\
072\end{array}$ & 0.0662 \\
\hline
\end{tabular}

ATumors were divided pathologically into two categories (see text for details).

${ }^{\mathrm{B}}$ Left side colon, including the ascending colon, sigmoid colon and rectosigmoid colon

CFor details of the extent of lymph node dissection, refer to text. However, the extent of D3 dissection is greater than that of D2 dissection.

A-colon, ascending colon; T-colon, transverse colon; $\mathrm{CI}$, confidence interval.

Table 3. Cox regression model analysis for prediction of tumor recurrence between 3 and 5 years after surgery

\begin{tabular}{|c|c|c|c|c|c|}
\hline Variables & & $\begin{array}{l}\text { Estimate } \\
\text { (SE) }\end{array}$ & $\begin{array}{l}\text { Hazard } \\
\text { ratio }\end{array}$ & $95 \%$ CI & $P$-value \\
\hline Node status & $\begin{array}{l}\text { Positive } \\
\text { (vs. } \\
\text { negative) }\end{array}$ & $\begin{array}{l}0.44691 \\
(0.16257)\end{array}$ & 1.563 & $1.137-2.150$ & 0.006 \\
\hline \multirow[t]{2}{*}{$\mathrm{T}$ factor } & $\begin{array}{l}\text { T3 (vs. } \\
\text { T2/T1) }\end{array}$ & $\begin{array}{l}0.49474 \\
(0.29657)\end{array}$ & 1.640 & $0.917-2.933$ & 0.095 \\
\hline & $\begin{array}{l}\text { T4 (vs. } \\
\text { T2/T1) }\end{array}$ & $\begin{array}{l}0.63042 \\
(0.31852)\end{array}$ & 1.878 & $1.006-3.507$ & 0.0478 \\
\hline Gender & $\begin{array}{l}\text { Male (vs. } \\
\text { female) }\end{array}$ & $\begin{array}{l}0.21993 \\
(0.16622)\end{array}$ & 1.246 & $0.900-1.726$ & 0.1858 \\
\hline Tumor grade $A$ & $\begin{array}{l}\text { Low (vs. } \\
\text { high) }\end{array}$ & $\begin{array}{l}0.79497 \\
(0.50769)\end{array}$ & 2.214 & $0.819-5.990$ & 0.1174 \\
\hline Complication & $\begin{array}{l}\text { Yes (vs. } \\
\text { no) }\end{array}$ & $\begin{array}{l}0.27161 \\
(0.20517)\end{array}$ & 1.312 & $0.878-1.962$ & 0.1856 \\
\hline $\begin{array}{l}\text { Lymph node } \\
\text { dissection }^{B}\end{array}$ & D2 (vs. D3) & $\begin{array}{l}0.55774 \\
(0.16253)\end{array}$ & 1.747 & $1.270-2.402$ & 0.0006 \\
\hline
\end{tabular}

ATumors were divided pathologically into two categories (see text for details). BFor details of the extent of lymph node dissection, refer to text. However, the extent of D3 dissection is greater than that of D2 dissection.

$\mathrm{CI}$, confidence interval.

In conclusion, the results of the present study support intensive surveillance within the first 3 years after surgical resection in Japanese patients with Stage II and Stage III colon cancer. A reduction in surveillance intensity may be justified in patients with T1/T2 Stage III and T3 Stage II colon cancer. Meanwhile, continuing with intensive surveillance beyond 3 years in patients with T4 colon cancer or T3/T4 node-positive colon cancer may be beneficial.

\section{Abbreviations}

HR: hazard rate; DFS: disease free survival; JFMC: Japanese Foundation for Multidisciplinary Treatment of Cancer.

\section{Supplementary Material}

Supplementary figures.

http://www.jcancer.org/v08p4057s1.pdf

\section{Acknowledgements}

This study was supported by the non-profit organization Epidemiological and Clinical Research Information Network (ECRIN) and the Japanese Foundation for Multidisciplinary Treatment of Cancer (JFMC).

\section{Funding}

The non-profit organization Epidemiological and Clinical Research Information Network (ECRIN) and the Japanese Foundation for Multidisciplinary Treatment of Cancer (JFMC).

\section{Competing Interests}

The authors have declared that no competing interest exists.

\section{References}

1. Fitzmaurice C, Dicker D, Pain A, et al. Global Burden of Cancer 2013. JAMA Oncol. 2015;1: 505-27.

2. Shah MA, Renfro LA, Allegra CJ, et al. Impact of Patient Factors on Recurrence Risk and Time Dependency of Oxaliplatin Benefit in Patients With Colon Cancer: Analysis From Modern-Era Adjuvant Studies in the Adjuvant Colon Cancer End Points (ACCENT) Database. J Clin Oncol. 2016; 34: 843-53.

3. Sakamoto J, Ohashi Y, Hamada C, et al. Efficacy of oral adjuvant therapy after resection of colorectal cancer: 5 -year results from three randomized trials. J Clin Oncol. 2004; 22: 484-92.

4. Nordlinger B, Sorbye $\mathrm{H}$, Glimelius $\mathrm{B}$, et al. Perioperative FOLFOX4 chemotherapy and surgery versus surgery alone for resectable liver metastases from colorectal cancer (EORTC 40983): long-term results of a randomised, controlled, phase 3 trial. Lancet Oncol. 2013; 14: 1208-15.

5. Folprecht $G$, Gruenberger $T$, Bechstein $W$, et al. Survival of patients with initially unresectable colorectal liver metastases treated with FOLFOX/cetuximab or FOLFIRI/cetuximab in a multidisciplinary concept (CELIM study). Ann Oncol. 2014; 25: 1018-25.

6. Steele SR, Chang GJ, Hendren S, et al. Practice Guideline for the Surveillance of Patients After Curative Treatment of Colon and Rectal Cancer. Dis Colon Rectum. 2015; 58: 713-25.

7. Sargent DJ, Wieand HS, Haller DG, et al. Disease-free survival versus overall survival as a primary end point for adjuvant colon cancer studies: individual patient data from 20,898 patients on 18 randomized trials. J Clin Oncol. 2005; 23: 8664-70.

8. Chauvenet M, Lepage $\mathrm{C}$, Jooste $\mathrm{V}$, et al. Prevalence of patients with colorectal cancer requiring follow-up or active treatment. Eur J Cancer. 2009; 45: 1460-5.

9. Meyerhardt JA, Mangu PB, Flynn PJ, et al. Follow-up care, surveillance protocol, and secondary prevention measures for survivors of colorectal cancer: American Society of Clinical Oncology clinical practice guideline endorsement. J Clin Oncol. 2013; 31: 4465-70.

10. Schmoll HJ Van Cutsem E Stein A, et al. ESMO Consensus Guidelines for management of patients with colon and rectal cancer. a personalized approach to clinical decision making. Ann Oncol. 2012; 23: 2479-516.

11. Weiser MR, Landmann RG, Kattan MW, et al. Individualized prediction of colon cancer recurrence using a nomogram. J Clin Oncol. 2008: 26:380-5.

12. Sadahiro S, Tsuchiya T, Sasaki K, et al. Randomized phase III trial of treatment duration for oral uracil and tegafur plus leucovorin as adjuvant chemotherapy for patients with stage IIB/III colon cancer: final results of JFMC33-0502. Ann Oncol. 2015; 26: 2274-80.

13. Watanabe M, Nishida $\mathrm{O}$, Kunii $\mathrm{Y}$, et al. Randomized controlled trial of the efficacy of adjuvant immunochemotherapy and adjuvant chemotherapy for colorectal cancer, using different combinations of the intracutaneous 
streptococcal preparation OK-432 and the oral pyrimidines 1-hexylcarbamoyl-5-fluorouracil and uracil/tegafur. Int J Clin Oncol. 2004; 9: 98-106.

14. Watanabe M, Kodaira S, Takahashi T, et al. Randomized trial of the efficacy of adjuvant chemotherapy for colon cancer with combination therapy incorporating the oral pyrimidine 1-hexylcarbamoyl-5-fluorouracil. Langenbecks Arch Surg. 2006; 391: 330-7.

15. Kodaira S, Kikuchi K, Kunii Y, et al. Postoperative adjuvant chemotherapy with mitomycin C and UFT for curatively resected rectal cancer. Results from the Cooperative Project No.7 Group of the Japanese Foundation for Multidisciplinary Treatment of Cancer. Int J Clin Oncol. 1998; 3: 357-64.

16. Maeda H, Oba K, Hamada C, et al. Hazard rate of tumor recurrence over time: a pooled-analysis of three clinical trials with fluoropyrimidine-based adjuvant chemotherapy for colorectal cancer achieved by the Japanese Foundation for Multidisciplinary Treatment of Cancer (JFMC). Ann. Cancer Res. Ther. 2016; 24: 60-61.

17. Japanese Research Society for Cancer of Colon and Rectum. General rules for clinical and pathological studies on cancer of the colon, rectum and anus. Jpn J Surg. 1983; 13: 557-73.

18. Japanese Society for Cancer of the Colon and Rectum. Japanese classification of colorectal carcinoma, 4th edn. Tokyo, Japan: Kanehara Ltd. 1985

19. Yamamoto S, Inomata M, Katayama H, et al. Short-term surgical outcomes from a randomized controlled trial to evaluate laparoscopic and open D3 dissection for stage II/III colon cancer: Japan Clinical Oncology Group Study JCOG 0404. Ann Surg. 2014; 260: 23-30.

20. Tsuji $Y$, Sugihara K. Adjuvant chemotherapy for colon cancer: the difference between Japanese and western strategies. Expert Opin Pharmacother. 2016; 17: $783-90$.

21. Yoshida M, Ishiguro M, Ikejiri K, et al. S-1 as adjuvant chemotherapy for stage III colon cancer: a randomized phase III study (ACTS-CC trial). Ann Oncol. 2014; 25: 1743-9. 